\title{
Vulnerability Analysis of Bus Failure in Power Grid
}

\author{
Xuncheng Huang ${ }^{1, \mathrm{a}}$, Huan Qi ${ }^{2, \mathrm{~b}}$, Xiaopan Zhang, ${ }^{3, \mathrm{c}}$, Lifang Lu ${ }^{2, \mathrm{~d}}$, Yangyu Hu, ${ }^{1, \mathrm{e}}$ \\ ${ }^{1 .}$ Henan Electric Power Company, Zhengzhou, 450052, China; \\ 2. Huazhong University of Science and Technology, Wuhan,430074, China \\ 3.Wuhan University of Technology, Wuhan,430070, China \\ ahxunc@126.com,, 'qihuanster@foxmail.com, czxp.whut@163.com, \\ dulifang186@126.com, `48342757@qq.com
}

\begin{abstract}
This paper analyzes the network features of power grid with buses' cascading failures based on the theories and methods of complex networks. And it concludes detailed vulnerability analysis of buses in Huazhong power grid under three forms of attacks (maximum load attack, maximum degree attack and random attack) .It provides technical means for the prevention of cascading failure .
\end{abstract}

Keywords-complex network; power grid; cascading failure of bus; power grid vulnerability

Power system faults take severe harm to the normal operation of society. The studies of power grid's vulnerability focus on the structure of power grid itself and the accumulation of risk energy caused by different kinds of outside interferences. Risk energy can spread in a power grid or between different ones, which makes energy accumulate and develop risks. When the disturbed energy is large enough, it will spread energy along the slits in the grid whose damping is the least. This kind of spread does so much damage that it may ravage the power grid in some cases.

In China and abroad, studies of vulnerability concentrate on the field of complex system. Haimes ${ }^{[1]}$

(2002) pointed out vulnerability was a multidimensional concept. Motter $^{[2][3]}$ (2002, 2004) built a network model for power grid. Paolo ${ }^{[4]}$ Crucitti ( 2004) proposed a simple model for cascading failure based on dynamic allocation of network traffic in complex networks. R. Kinney ${ }^{[5]}$

(2005) took north American power grid for example to analyze the load and overload transmission substations . Sun $\mathrm{Ke}^{[6]}(2008)$ introduced complex network efficiency model and an efficiency assessment method . Li Tao, Fan Xingfang and $\mathrm{Xu}$ Xialing ${ }^{[7]}(2010)$ summarized current status of vulnerability assessment from two aspects: the vulnerability of state and structure. And they pointed out the trend of research. Yang Wenhui ${ }^{[8]}$ (2012) proposed key branches recognition method based on survivability assessment of power grid. Cai Zexiang, Wang Xinghua and Ren Xiaona ${ }^{[9]}$ reviewed the research progress and applications while complex network theory was applied in modeling topology of power grid. Liu Yang, Zhang Yan and Yang Zenghui ${ }^{[10]}$ ( 2012 ) put forward the risk assessment index system for power grid's cascading failures. Guidong Zhang ${ }^{[11]}$ ( 2013 ) analyzed the mechanism why cascading failures happened in Indian power grid . Jianwei Wang ${ }^{[12]}$ ( 2013 ) studied the effectiveness of the optimal protection strategy constructed in the scale-free network and power grid .

The paper analyzes buses' vulnerability under three forms of attacks(maximum load attack, maximum degree attack and random attack) based on the theory and method of complex network, which provides a new perspective for the research of grids' security and the cascading failure.

\section{The AbSTRACTION OF POWER GRID FROM COMPLEX NETWORK PERSPECTIVE}

Considering the features and characteristics of power grid, in order to simply analysis while building complex network model of power grid, some abstract rules are formulates as follow:

(1) Only consider electric power transmission network, and ignore the influence of the distribution network;

(2) Only consider power plant, $220 \mathrm{kv}, 550 \mathrm{kv}$ substations, the main buses in distribution network and the branches between them. All of the buses with different voltages in the same substation will be combined;

(3) Combining the transmission lines on the same tower and do not include them in the parallel capacitor branches, which means cancelling the rings and multiple edges in topology model of power grid and making the corresponding graph simpler;

(4) Transmission lines and transformer branch are edges in topological model of power grid and all edges are undirected. The topological structure of Huazhong power grid is shown in figure 1.

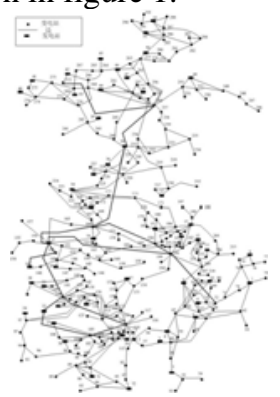

Figure 1 The Topological StRUCtURE OF POWER GRID

Table 1 describes the topological index of Huazhong 
power grid. It includes 295 buses and 413 branches. The number of power plant is 52. The average degree of branch is 2.800 .

Table1 The Topological Features of Huazhong Power GRID

\begin{tabular}{|c|c|c|c|c|c|c|c|}
\hline $\begin{array}{c}\text { Popolcegcal modexes } \\
\text { Infrastructure }\end{array}$ & N & E & G & K & C & L & B \\
\hline Pomer Grid & 295 & 413 & 52 & 2.800 & 0.096 & 7922 & 2035 \\
\hline
\end{tabular}

(Where $\mathrm{N}$ represents the number of buses, $\mathrm{E}$ represents the number of branches, $G$ is the number of power plants, and $<\mathrm{k}>$ represents the average degree of buses, $\mathrm{C}$ is average clustering coefficient, $\mathrm{L}$ represents characteristic path length and $B$ is the average betweenness of buses.)

The average degree is a very important statistical indicator of complex network and it means the average degree of all nodes in a network ( $k=\frac{1}{N} \sum_{i=1}^{N} k_{i j}$ ). The larger this number, the connections between nodes are more probably existing and the number of connections in network is larger. The average path length of this network is 7.922, while the average clustering coefficient is 0.0961. For one determined network, supposing the power exchange and spread along with the shortest path between buses, then the betweenness of buses can indicate the load they assume. The betweenness of Huazhong power grid is 2035.

\section{The Design of Bus Cascading Failure Model FOR POWER SYSTEM}

The Motter model is used to build power system load model. Motter model is proposed by scholar s of complex network. They tried to study the relationship between structure and function of power grid by this model. The detailed process is described here:

(1)Suppose the power exchange and spread along with the shortest paths between buses;

(2)The load of bus is defined as the number of shortest paths through it and that is the betweenness of bus;

(3)The load can't exceed the capacity of a bus. If one bus's load exceed its capacity, then the bus fails and should be removed from this power grid and branches connected with this bus will be removed too;

(4)If a bus is removed, the load of every bus should be redistributed until the power grid achieves new balance.

Based on Motter model, the model of power grid is built as follows. If bus $\mathrm{i}$ and bus $\mathrm{j}$ are in the power grid, $(\mathrm{i}, \mathrm{j})$ means the shortest path between these two buses. And for any bus $\mathrm{k}$ in power grid,

$L_{k}^{(i, j)}$ represents whether the shortest path between bus $\mathrm{i}$ and bus $\mathrm{j}$ gets through bus $\mathrm{k}$. If the shortest path gets through bus $\mathrm{k}$, the value of $L_{k}^{(i, j)}$ is 1. On the contrary its value is 0 . So the number of shortest paths getting through bus $\mathrm{k}$ can be described as $^{L_{k}=\sum_{i, j} L_{k}^{(i, j)}} . L_{k}$ is the load of bus $\mathrm{k}$ when it operates normally. If the maximum load of bus $\mathrm{k}$ is ${ }^{C_{k}}$. There is $C_{k}=(1+\alpha) L_{k}, k=1,2,3 \cdots N$, where constant $\alpha \geq 0$ represents redundant capacity coefficient of bus. If the load of $k$ exceeds the maximum load $C_{k}$. The bus $\mathrm{k}$ will be removed from power grid because of over loading.

\section{The Design of Buses’ ATtACK Form IN POWER GRID}

In order to measure the vulnerability of buses cascading failure under different perturbation strategies, three forms of bus attacks defined in complex network are studied and they are improved to be three hazard risk models of power grid:

(1)Random attack due to unpredictable factors. Power system will be affected by the harms from rapid climate change in nature or man-made faults at any time. In random perturbation, every bus has the same probability to be removed.

(2)Maximum degree attack considering the connection of grid. In this kind of attack, the bus whose degree is the largest will be removed firstly.

(3)Maximum load attack of power grid. The branches in power grid are used to transmit energy.

\section{The Simulation ANALysis of HuazhONG POWER GRID}

Based on the analysis above, the steps of simulation are as follows:

(1)The structure matrix of power grid is given firstly. Suppose that power grid operates normally under the initial condition, the load of bus $\mathrm{k}$ is $L_{k}=\sum_{i, j} L_{k}^{(i, j)}$, that is the betweenness of it, the capacity of bus $k$ is $C_{k}=(1+\alpha) L_{k}, k=1,2,3 \cdots N$. Once the load exceeds the capacity of a bus, it will be removed from power grid.

(2)Attack the power grid according to pre-set form and remove the bus which is attacked.

(3)Based on bus cascading failure model for power system, the load will be redistributed in power grid. If the load of any bus exceeding its capacity, this bus should be removed. The load of power grid will be redistributed again. Repeat this step until the power grid achieves a new balance.

(4)Check whether the number of removed buses has reached the preset. If yes, simulation is end. And if not, return to the second step.

The figure 2 shows the change of network efficiency with the removal ratio of buses.

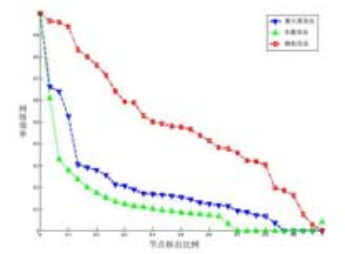

Figure 2 CHANGE OF NETWORK EFFICIENCY WITH THE REMOVAL RATIO OF BUSES 
It can be learned from figure 2, as the increasing of the removal ratio of buses, the network efficiency decreases significantly. That's because connections between buses decrease when the number of removed buses increases. And buses should get through more branches to be connected. The path length between buses increases significantly, so the network efficiency decreases significantly too. In addition, the rates of decline of network efficiency are different with different forms of attacks. The rate of decline is fastest in maximum load attack, the secondly fast is maximum degree attack, while the random attack is slowest.

In order to research initial disturbance's influence on power grid with different capacity factors, the avalanche scale $\delta$ of bus is defined as: $\delta=1-N^{*} / N$. Where $N$ means the number of normally operated buses before the cascading failure happened, while $N^{*}$ is the number of normally operated buses after cascading failure happened. So the avalanche scale of buses indicates the damage rate of them. In maximum load attack and maximum degree attack, every time five buses are removed and the avalanche scale of grid is calculated when the power grid achieves a new balance after cascading failure happened. It has been shown in figure 3 .

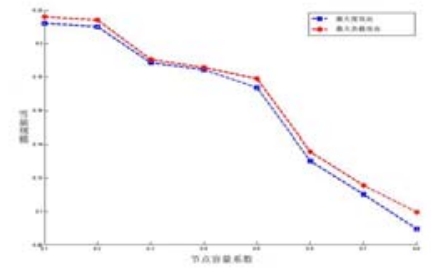

Figure 3 The Change of AVALANCHE SCALE OF GRID ALONG WITH DIFFERENT CAPACITY FACTORS UNDER DIFFERENT FORMS OF ATTACKS

As shown in figure 3, if the capacity factor is small, every form of attack can cause big avalanche scale, while the capacity factor is big, the avalanche scale is smaller and smaller and in this case, the connection performance of grid is better. So it's necessary to make a balance between functional properties and economic costs.

Through the study of removal order of buses, and in that order, we can get the paths which risk energy spread along with. That is the slit mentioned above. Power grid is a man-made complex system which can be controlled. The targeted design of locations to collect information, information transferring, the order of information processing and priority is very valuable for engineering application of the power grid.

\section{ACKNOWLEDGEMENTS}

This work was financially supported by the National Natural Science Fund(60904073) and The HubeiNatural Science Fund(2012FFB05111)

\section{REFERENCES}

[1] Haimes, Y.Y., Longstaff, T.,. The Role of Risk Analysis in the Protection of Critical Infrastructures Against Terrorism. Risk Analysis. 2002, 22(3):439-444

[2] Motter A, Nishikawa T, Lai Y. . Cascade-based attacks on complex networks. Physical Review E. 2002, 66:065102(1-4)

[3] Motter A. Cascade Control and Defense in Complex Networks. Physical Review Letter. 2004, 93: 098701 (1-4)

[4] Paolo Crucitti, Model for cascading failures in complex networks Physical Review E, 69, 045104(R), 2004

[5] R. Kinney, Modeling cascading failures in the North American power grid, The European Physical Journal B, 46, 101-107, 2005

[6] sun can, some applied research in theory of complex networks in power grid, Ph.D. Thesis, zhejiang university, 2008

[7] Lin tao; Fan Xing yuan; Xu Xialing, power system vulnerability assessment method research review, journal of electric power science and technology, 2010, 25(4)

[8] Yang Wenhui, prevent the chain tripping the key line backup protection and emergency control strategy research, north China electric power university, Ph.D. Thesis, 2012

[9] Cai Zexiang; Wang Xinghua; Ren Xiaona, complex network theory and its application in electric power systems research review, the grid technology in 2012, 36 (11)

[10] LiuYang; Zhang Yan; Yang Zenghui; And so on, the chain transmission system fault risk evaluation index and calculation method of the modern electric power in 2012, 29 (4)

[11] Guidong Zhang, Understanding the cascading failures in Indian power grids with complex networks, Physica A, 392, 3273-3280, 2013

[12] Jianwei Wang, Robustness of complex networks have the local protection strategy against cascading failures, Safety Science, 53, 219-225, 2013 